\title{
Rarefaction Throttling Effect: Influence of the Bend in Micro-Channel Gaseous Flow
}

\author{
Wei Liu, ${ }^{1}$ Guihua Tang, ${ }^{1}$, a) Wei Su, ${ }^{2}$ Lei Wu, ${ }^{2}$ Yonghao Zhang ${ }^{2}$ \\ ${ }^{1}$ MOE Key Laboratory Thermo-Fluid Science and Engineering, School of Energy and Power Engineering, Xi'an \\ Jiaotong University, Xi'an 710049, China \\ ${ }^{2}$ James Weir Fluids Laboratory, Department of Mechanical and Aerospace Engineering, University of Strathclyde, \\ G1 1XJ Glasgow, United Kingdom
}

a) Author to whom correspondence should be addressed: ghtang@mail.xjtu.edu.cn

Micro-bends are frequently encountered in micro-electro-mechanical systems (MEMS) as a basic unit of complex geometry. It is essential for a deep understanding of the rarefied gas flow through bent channel. In this paper, a two-dimensional pressure-driven gas flow in a micro-channel with two bends is investigated by solving the Bhatnagar-Gross-Krook kinetic equation via the discrete velocity method in the slip and transition flow regimes. The results show that the mass flow rate (MFR) through the bent channel is slightly higher than that in the straight channel in the slip flow regime but drops significantly as the Knudsen number increases further. It is demonstrated that the increase of MFR is not due to the rarefaction effect but to the increase in cross-section of the bent corners. As the rarefaction effect becomes more prominent, the low-velocity zones at the corners expand and the gas flow is "squeezed" into the inner corner. The narrowed flow section is similar to the throttling effect caused by the valve, and both the changes in MFRs and the pressure distribution also confirm this effect. The classical Knudsen minimum changes due to this "rarefaction throttling effect". The Knudsen number at which the minimum MFR occurs gradually increases with the bend angle, and finally disappears in the transition flow regime. In addition, the onset of rarefaction throttling effect shifts to a smaller Knudsen number with a lower tangential momentum accommodation coefficient.

\section{INTRODUCTION}

Micron-sized devices fabricated by micro-electronic fabrication processes are the major components in micro-electromechanical system (MEMS), including microsensors, micro-turbines, and micro-fuel cells. ${ }^{1,2}$ Due to the features of miniaturization and sophistication, typical micro-device often contains high-density microchannel networks ${ }^{3,4}$, which are composed of hundreds to thousands of channels. The typical length scale of these microchannels ranges from a few hundred to a few microns, sometimes even to sub-micron. Due to the design limitation, channels with bends are encountered in most of the miniaturized devices. For instance, micro heat exchanger generally uses a serpentine channel containing many bends to keep the device efficient and compactable. ${ }^{5}$ Therefore, the study of gas flows through complex micro-channels has great practical importance. Over the years, systematic understanding of internal rarefied gas flows has attracted attention in both experimental and theoretical 
research. ${ }^{6-9}$ So far, gas flows in straight microchannels have been investigated extensively. However, the research work for the flow in channels with bends, to the authors' best knowledge, is very limited. As the emergence of bend could lead to some new and counter-intuitive phenomena, e.g., the mass flow rate (MFR) might increase in bent channels ${ }^{5,10-12}$, a detailed investigation on the bending effect in gas flow through micro-channels would be significantly helpful for the design of MEMS.

In most of the miniaturized devices, the mean free path of gas molecules $\lambda$ is of the same order as or even larger than the system characteristic flow length $H .{ }^{1}$ When $H$ decreases to significantly less than $\lambda$, the ratio of these two lengths, which is defined as the Knudsen number $(\mathrm{Kn}=\lambda / H)$, may become relatively large. Under such circumstances, theories derived from the continuum assumption including the conventional Navier-Stokes equations do not work. ${ }^{13}$ This is due to the fact that, the shear stress and heat flux in the fluid dynamic models cannot be simply expressed in terms of the lower-order macroscopic quantities. A number of rarefied effects have been revealed in rarefied gas flows, including the velocity $\operatorname{slip}^{14}$, temperature jump ${ }^{15}$, Knudsen paradox ${ }^{16}$, and thermal transpiration ${ }^{17}$. However, how the complex structures in micro-channel affect the gas flow remains less investigated. By using the integrated pressure sensors, experiments of gas flow in microchannels with complicated geometries have been reported in literature ${ }^{[18-20]}$. Lee et al. investigated the MRFs and the pressure distributions in three different bend configurations including the miter, curved and double-bent microchannels. ${ }^{18}$ Their results showed that additional pressure drops are induced by bends and the MFR in double-bent channel is reduced to about $90 \%$ of the one in the straight channel. Varade et al. conducted experiments for nitrogen gas flows through a tube with a single bend in the continuum flow and early slip regimes $(0.0003<\mathrm{Kn}<0.0385) .{ }^{20}$ It was found that the flow separation near the corner causes a large pressure drop at small Knudsen numbers while the influence gradually decays as the Knudsen number increases. However, these works are limited to the flow regimes with small Knudsen numbers. Besides, to measure the small MFR of highly rarefied low-speed gas flow, a large pressure ratio is necessary to drive the gas flow, which makes it expensive and difficult to conduct an experiment in incompressible limit.

Due to the constraint in experiments, efficient numerical simulation for gas flows at micro-scale has received widespread attention and been improved with significant progress. When the Navier-Stokes equations break down, the Boltzmann equation for the velocity distribution function (VDF) of gas molecules, which is derived based on the gas kinetic theory, is a fundamental way to describe the rarefied gas dynamics. ${ }^{21}$ The Direct Simulation Monte Carlo (DSMC) method ${ }^{22}$ is widely used for the numerical solution of the Boltzmann equation. By using a set of simulated particles each of which could represent a great number of real gas molecules, the DSMC method phenomenologically mimics the streaming and binary collisions of molecules, as well as the gas-boundary interactions in the computational domain. It has been applied to investigate the MFRs and the pressure distributions in bent channels under different Mach numbers and Reynolds numbers ${ }^{12,23,24}$, where the flow field and the pressure distribution along the bend are found to be similar to those in the straight channel, except for some differences near the corner of the bend. Although White et al. ${ }^{12}$ attributed such discrepancies to the change of rarefied effect, Rovenskaya ${ }^{5}$ showed that the differences are still present with fixed Knudsen number and gradually become significant when the Mach number increases. This indicates 
that the local influence of the bend is due to the gas compressibility. Besides, when both the total length and the pressure difference between inlet/outlet are the same, it is found that the MFR along the bent channel is slightly higher than that in the straight channel in near-continuum flow regime ${ }^{12}$ but the difference disappears when the Knudsen number increases ${ }^{10,11}$.

Although the DSMC method has been successfully applied to a wide range of problems in rarefied gas dynamics, the statistical feature of the particle method makes it very inefficient in simulating low-speed flows ${ }^{25}$, which is frequently encountered in micro-systems. When gas velocity is far smaller than the most probable speed of gas molecules, the deterministic numerical simulation approaches relaying on the discrete velocity method (DVM) are attractive alternatives to solve the Boltzmann equation. ${ }^{26}$ These methods adopt a set of discrete velocities to represent the continuous molecular velocity space. Then the VDFs which are discrete in the velocity space but continuous in the spatial space are solved by conventional computational fluid dynamic methods. ${ }^{27-29}$ Generally speaking, for the solution of highly-rarefied gas flow, a large number of discrete velocities is required in order to capture non-equilibrium effects precisely. ${ }^{30,31}$ In practice, deterministic solution is commonly sought for the gas kinetic models that reduce the complicated collision operator in the Boltzmann equation to a simpler collision relaxation term; one frequently used is the Bhatnager-Gross-Krook (BGK) kinetic equation ${ }^{32}$, which has been proven to be sufficiently accurate on many fundamental issues such as gas slider bearing problem ${ }^{33}$, Knudsen paradox ${ }^{34}$, etc.

One of the interesting problems in rarefied gas dynamics, i.e. the Knudsen minimum or Knudsen paradox, draws attention for years due to its important applications to the micro-machines and other devices with tiny air gap. ${ }^{35,36}$ This phenomenon was firstly observed by Knudsen et al. ${ }^{37}$ in experiments of Poiseuille flow driven by the identical pressure drop in the channels with varying widths. Although it has been extensively studied in both experimental and theoretical analyses, the research that focuses on how the geometry of the microchannels affect the gas flow is quite limited. Sharipov et al. ${ }^{38}$ investigated the rarefied gas flow through a zigzag channel by using the linearized kinetic equation and found that the aspect ratio of the channel affects MFRs while the underlying reason behind it is still unknown. Besides, the effect of accommodation coefficient of the bounding surfaces, which could affect the MFR significantly ${ }^{39}$, awaits to be studied under complex geometries. As a benchmark of the gas flow problem in complex geometry, the gas flow in a bent channel is a good starting point to help us seek for fundamental understanding of flow mechanism.

Therefore, the aim of this paper is to investigate the effect of the bend on the behavior of a pressure-driven flow in the slip and transition flow regimes by using deterministic solutions of the linearized BGK equation. The geometry of the bent channel is introduced in Sec. II, and the linearized BGK equation as well as the numerical scheme is briefly described in Sec. III. The influences of the bend on the pressure-driven gas flow and the corresponding mechanisms are discussed in Sec. IV. Finally, conclusions are given in Sec. V.

\section{FORMULATION OF THE PROBLEM}

As shown in FIG. 1, we consider a two-dimensional pressure-driven gas flow through a micro-channel with two bends in a Cartesian coordinate system $\mathbf{x}(x, y)$. The micro-channel is divided 
into three segments equally: the front and rear segments of the channel are horizontal, while the middle one connected to the other two parts by corners is located with a sloping direction. The length of each part is $L / 3$, where $L$ is the total flow length and $L$ ' is the channel length in the $x$ direction. The bend angle $\theta$, which is defined as the angle between the direction of the middle segment and the $x$ direction, varies from $0^{\circ}$ to $90^{\circ}$. To easily implement boundary condition in the Cartesian coordinates, the stair-step grid method is used to approximate the inclined channel walls. The width of the channel $H=1$. The ratio of the total length $L$ to the width of the channel $H$ sets to 13.5 while the solid surfaces of the channel extend to infinity in the direction perpendicular to the $x-y$ plane, so the problem is effectively two-dimensional. ${ }^{5,40-42}$ Due to the fact that the material of silicon, which is commonly used for micron-sized devices, is a good heat conductor, the gas flow in the micro-channel could be considered isothermal. ${ }^{13}$ In this work, the gas flow is driven by the pressure difference between the inlet/outlet of the channel and we are interested in the influence of bend on the MFR at various Knudsen numbers.

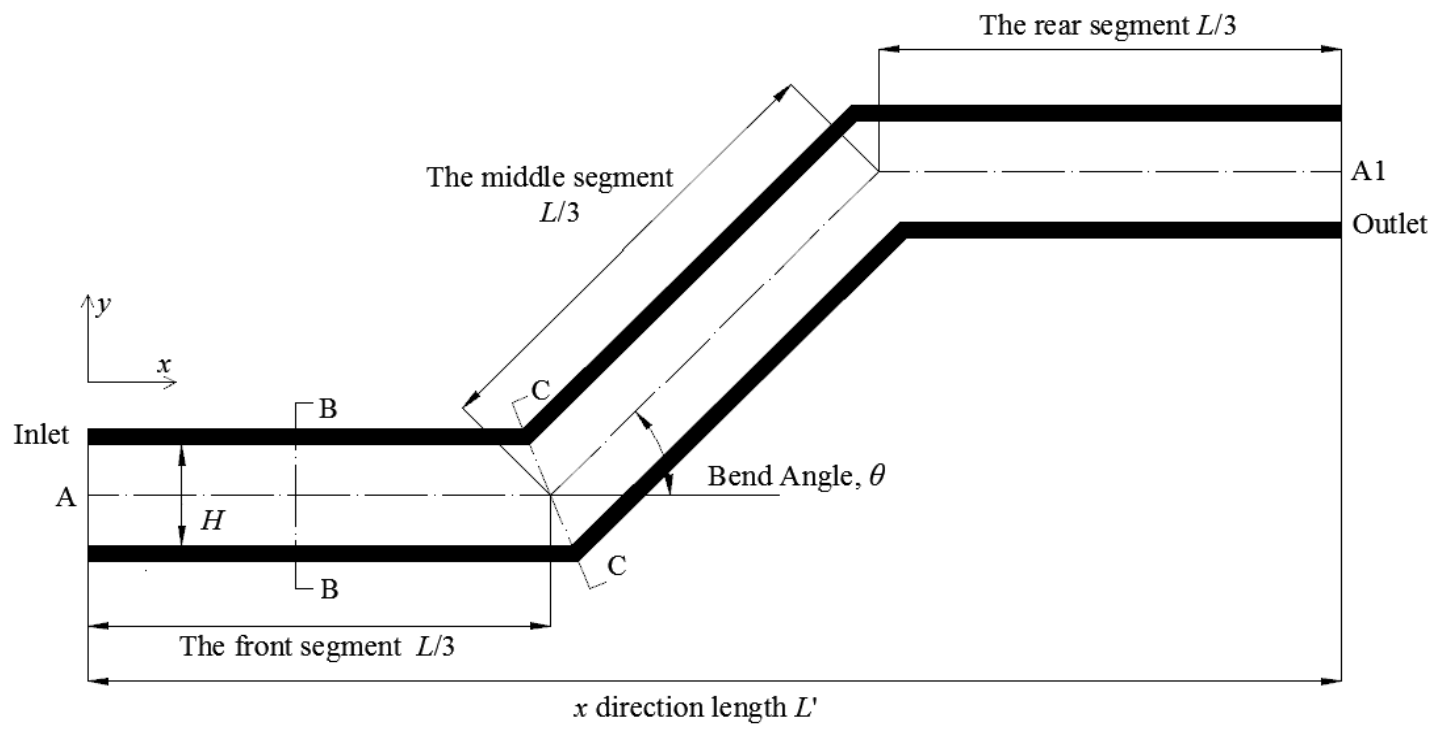

FIG. 1. Schematic of a two-dimensional micro-channel with two bends. The dashed line A-A1 represents the centerline of the microchannel, while the cross-sections B-B and C-C represent sections away from the corner and at the corner, respectively.

\section{GAS KINETIC THEORY AND NUMERICAL SCHEME}

\section{A. The gas kinetic theory}

The Boltzmann equation, which describes the evolution of the VDF in dependence of the molecular velocity, spatial position, and time evolution, is valid for the entire range of Knudsen numbers. In this paper, we use the following form of BGK equation instead of the full Boltzmann equation. ${ }^{32}$ The BGK equation in the two-dimensional spatial space and the two-dimensional velocity space could be written in Cartesian coordinate as

$$
\frac{\partial f}{\partial t}+\mathbf{v} \cdot \frac{\partial f}{\partial \mathbf{x}}=\frac{p}{\mu}\left(f_{e q}-f\right)
$$




$$
f_{e q}=\frac{\rho_{0}}{2 \pi R T} \exp \left(-\frac{(\mathbf{v}-\mathbf{u})^{2}}{2 R T}\right),
$$

where $f(t, \mathbf{x}, \mathbf{v})$ denotes the VDF, $\mathbf{v}=\left(v_{x}, v_{y}\right)$ the molecular velocity, $\mathbf{x}=(x, y)$ the spatial coordinate, $p$ the gas pressure, $\rho_{0}$ the reference density and $\mu$ the shear viscosity of the gas. Note that physically, the VDF is defined in the three-dimensional molecular velocity space, however, for isothermal problems, the VDF can be reduced to two-dimensional velocity space. We have tested that this does not affect the flow velocity or shear stress. ${ }^{34}$

For the numerical solution of the system, it is convenient to normalize all the variables and function. Without losing generality, the following non-dimensional variables are defined as

$$
\begin{aligned}
& \mathbf{x}=\frac{x}{H}, \hat{\imath} \quad H / \sqrt{\frac{2 R T_{0}}{2}}, \quad \mathbf{u}=\frac{\mathbf{u}}{\sqrt{2 R T_{0}}}, \quad \mathbf{v}=\frac{\mathbf{v}}{\sqrt{2 R T_{0}}}, \\
& \rho=\frac{\rho}{\rho_{0}}, p=\frac{p}{p_{0}}, \Delta p=\frac{\Delta p}{p_{0}}, T=\frac{T}{T_{0}}, f=\frac{f}{\rho_{0} / 2 R T_{0}},
\end{aligned}
$$

where $\mathbf{u}=\left(u_{x}, u_{y}\right)$ is the macroscopic velocity, $R$ is the specific gas constant, $T$ is the gas temperature, $T_{0}$ is reference temperature and $H$ is the characteristic flow length. Note that the symbol hat denotes the dimensionless value in this paper. The Knudsen number is defined as

$$
\mathrm{Kn}=\frac{\lambda}{H}=\frac{\mu\left(\mathrm{T}_{0}\right)}{\bar{p} H} \sqrt{\frac{\pi R T_{0}}{2}},
$$

where $\lambda$ is the mean free path of the gas molecules, which is related to its shear viscosity $\mu$ and the average pressure $\bar{p}$ at the reference temperature. Then, the non-dimensional form of the BGK equation and the Maxwell velocity distribution function becomes

$$
\begin{gathered}
\frac{\partial f}{\hat{\partial}_{.}} \perp \mathbf{v} \cdot \frac{\partial f}{\ldots}=\frac{\sqrt{\pi}}{2 \mathrm{Kn}}\left(f_{e q}-f\right), \\
f_{e q}=\frac{\rho}{\pi T} \exp \left(-\frac{(\mathbf{v}-\mathbf{u})^{2}}{T}\right) .
\end{gathered}
$$

Macroscopic quantities of the gas flow could be obtained from the velocity moments of the velocity distribution function. Thus, the non-dimensional forms of density, velocity and temperature are calculated as below

$$
\begin{gathered}
\rho=\int f d \mathbf{v}, \\
\rho \mathbf{u}=\int \mathbf{v} f d \mathbf{v}, \\
\rho T=\frac{2}{3} \int(\mathbf{v}-\mathbf{u})^{2} f d \mathbf{v} .
\end{gathered}
$$

Equation (5) must be combined with the boundary condition that determines the interaction of gas/surface when gas molecules collide with the wall surface. Here, the Maxwellian diffuse reflection boundary condition is used. ${ }^{43}$ Suppose that the solid surface is static with a temperature of $T_{0}$, for 
$\mathbf{v} \cdot \mathbf{n}>0$ (n represents the outward unit normal vector of the wall) the VDF for the molecules reflected by the wall is written as

$$
f(\mathbf{x}, \mathbf{v})=\alpha \frac{\rho_{w}}{\pi} \exp \left(-|\mathbf{v}|^{2}\right)+(1-\alpha) f(\mathbf{x}, \mathbf{v}-2[\mathbf{v} \cdot \mathbf{n}] \mathbf{n}),
$$

where $\alpha$ represents the tangential momentum accommodation coefficient (TMAC). ${ }^{44}$ This boundary condition assumes that, when colliding with solid surface, $1-\alpha$ parts of the molecules are specularly reflected while the rest are diffusively reflected. Purely diffuse or specular reflection corresponds to the cases of $\alpha=1$ and 0 , respectively. $\rho_{w}$ is given by

$$
\rho_{w}=2 \sqrt{\pi} \int_{\mathbf{v}^{\prime} \cdot \mathbf{n}<0}\left|\mathbf{v}^{\prime} \cdot \mathbf{n}\right| f\left(\mathbf{x}, \mathbf{v}^{\prime}\right) d \mathbf{v}^{\prime}
$$

to guarantee that the mass flux across the solid surface equals to zero.

\section{B. The linearized BGK equation}

When the pressure gradient is small, namely $|L \Delta p / p d x|<<1$, and the gas flow velocity is much smaller than the most probable molecular speed $v_{m}=\sqrt{2 R T_{0}}$ in micro-flows, the VDF $f$ could be linearized about the global equilibrium state $E_{e q}$ as follows:

$$
f=E_{e q}(1+h), E_{e q}=\exp \left(-|\mathbf{v}|^{2}\right) / \pi,
$$

where the perturbation VDF $h(t, \mathbf{x}, \mathbf{v})$ is governed by the following linearized BGK equation: ${ }^{38,45}$

$$
\begin{gathered}
\frac{\partial h}{\partial t}+\mathbf{v} \cdot \frac{\partial h}{\partial \mathbf{x}}=\mathrm{L}(\tilde{\mathrm{n}}, \mathbf{u}, \tau)-\frac{\sqrt{\pi}}{2 \mathrm{Kn}} h, \\
\mathrm{~L}(\tilde{\mathrm{n}}, \mathbf{u}, \tau)=\frac{\sqrt{\pi}}{2 \mathrm{Kn}}\left[\tilde{\mathrm{n}}+2 \mathbf{u} \cdot \mathbf{v}+\tau\left(|\mathbf{v}|^{2}-\frac{3}{2}\right)\right],
\end{gathered}
$$

where the perturbed number density, the flow velocity, and the perturbed temperature are calculated as follows:

$$
\begin{gathered}
\tilde{\mathrm{n}} \equiv \rho-1=\int h E_{e q} d \mathbf{v}, \\
\rho \mathbf{u}=\int \mathbf{v} h E_{e q} d \mathbf{v}, \\
\tau=T-1=\frac{2}{3} \int(\mathbf{v})^{2} h E_{e q} d \mathbf{v}-\tilde{\mathbf{n}} .
\end{gathered}
$$

The linearized form of the gas kinetic boundary conditions is given below. The periodic-pressure-driven boundary conditions are applied along the $x$ direction at the inlet $(x=0)$ and outlet $\left(x=L^{\prime}\right):^{38}$

$$
\left.\begin{array}{c}
h\left(0, y, v_{x}, v_{y}\right)=B+h\left(L^{\prime}, y, v_{x}, v_{y}\right), \text { when } v_{x}>0 \\
h\left(L^{\prime}, y, v_{x}, v_{y}\right)=-B+h\left(0, y, v_{x}, v_{y}\right), \text { when } v_{x}<0
\end{array}\right\}
$$

where $B$ is perturbed pressure constant and $\Delta p=\int B E_{e q} d \mathbf{v}$ is perturbed pressure difference. 
While on the upper and lower solid surfaces, the linearized form of diffuse-specular boundary condition is written as ${ }^{43}$

$$
\begin{gathered}
h(\mathbf{x}, \mathbf{v})=\frac{2 \alpha}{\sqrt{\pi}} \int_{\mathbf{v}^{\prime}: \mathbf{n}<0}\left|\mathbf{v}^{\prime} \cdot \mathbf{n}\right| h\left(\mathbf{x}, \mathbf{v}^{\prime}\right) \exp \left(-\left|\mathbf{v}^{\prime}\right|^{2}\right) d \mathbf{v}^{\prime} \\
+(1-\alpha) h(\mathbf{x}, \mathbf{v}-2[\mathbf{v} \cdot \mathbf{n}] \mathbf{n}) .
\end{gathered}
$$

When the gas flow reaches the steady state, the dimensionless MFRs of the pressure-driven flow are calculated by $Q=\int_{0}^{1} \rho u_{x} / \Delta p d y=Q_{0} /\left(\sqrt{2 R T} \rho_{0} \Delta p H\right)$ where $Q_{0}=\int_{0}^{H} \rho u_{x} d y$.

\section{DVMC. The discrete velocity method}

In the DVM, the molecular velocity space $\mathbf{v}$ is represented by a set of discrete velocities. In this work, $N_{v}$ non-uniform points are used to discretize the molecular velocity in each direction, ${ }^{30}$

$$
v_{x, y}=\frac{v_{\max }}{\left(N_{v}-1\right)^{3}}\left(-N_{v}+1,-N_{v}+3, \ldots, N_{v}-1\right)^{3},
$$

where the discrete velocities are distributed in a square of $[-4,4]^{2}$ with $v_{\max }=4$, and are refined near $v_{x, y}=0$. This method of choosing the discrete velocities rather than the Gauss-Hermite quadrature has been proven efficient in simulations of micro/nano-scale gas flow, especially in capturing the rapid variations in the VDF at larger Knudsen numbers. ${ }^{34}$ Finally, Eq. (13) is solved by the following iterative method

$$
\frac{\sqrt{\pi}}{2 \mathrm{Kn}} h^{(j+1)}+\mathbf{v} \cdot \frac{\partial h^{(j+1)}}{\partial \mathbf{x}}=\mathrm{L}\left(\tilde{\mathrm{n}}^{(j)}, \mathbf{u}^{(j)}, \tau^{(j)}\right)
$$

where the scripts $(j)$ and $(j+1)$ denotes two consecutive iteration steps. Here, we omitted the derivation with respect to the time since we are only interested in the steady-state solution. The spatial derivatives are approximated by a second-order upwind finite difference scheme, and the iteration is terminated when the relative error of the macroscopic velocity $\mathbf{u}$ between two consecutive iteration steps is less than $10^{-10}$.

\section{Multiple-Relaxation-Time lattice Boltzmann method}

Because the spatial mesh is required to be smaller than the mean free path, the DVM becomes time-consuming in the near continuous flow area.,34 For comparison, we use the Multiple-Relaxation-Time lattice Boltzmann method (MRT-LBM) ${ }^{46}$ with zero velocity-slip boundary condition to simulate gas flow in the continuum flow regime. Under the assumption that the flow speed is small, the MRT-LBM is proven to be equivalent to solving the Navier-Stokes equations in the near-incompressible limit for single phase flows through complex geometries ${ }^{47}$, and has been extended for multiphase flow simulation successfully ${ }^{48,49}$. The two dimensional-nine-velocity (D2Q9) LBM with a multiple-relaxation-time collision operator can be written as: ${ }^{50,51}$ 


$$
f_{i}\left(t+\delta, x+v_{i} \delta_{t}, v_{i}\right)-f_{i}\left(t, x, v_{i}\right)=-\left(\mathbf{M}^{-1} \mathbf{S M}\right)_{i j}\left[f_{j}-f_{e q, j}\right] .
$$

Here $\left\{f_{i}\left(t, x, v_{i}\right): i=0,1, \ldots, 8\right\}$ are the discrete distribution VDF at time $t$ and position $x$ associated with the discrete velocities $\left\{v_{i}: i=0,1, \ldots, 8\right\}$ defined by $v_{0}=(0,0), v_{1}=-v_{3}=(1,0) c$, $v_{2}=-v_{4}=(0,1) c, \quad v_{5}=-v_{7}=(1,1) c$ and $v_{6}=-v_{8}=(-1,-1) c$, where $c=\delta_{x} / \delta_{t}$ with $\delta_{x}$ being the lattice spacing and $\delta_{t}$ being the time step. $\mathbf{M}$ is a $9 \times 9$ invertible transformation matrix projecting the discrete $\mathrm{VDF} f_{i}$ onto the moment space:

$$
\mathbf{M}=\left(\begin{array}{ccccccccc}
1 & 1 & 1 & 1 & 1 & 1 & 1 & 1 & 1 \\
-4 & -1 & -1 & -1 & -1 & 2 & 2 & 2 & 2 \\
4 & -2 & -2 & -2 & -2 & 1 & 1 & 1 & 1 \\
0 & 1 & 0 & -1 & 0 & 1 & -1 & -1 & 1 \\
0 & -2 & 0 & 2 & 0 & 1 & -1 & -1 & 1 \\
0 & 0 & 1 & 0 & -1 & 1 & 1 & -1 & -1 \\
0 & 0 & -2 & 0 & 2 & 1 & 1 & -1 & -1 \\
0 & 1 & -1 & 1 & -1 & 0 & 0 & 0 & 0 \\
0 & 0 & 0 & 0 & 0 & 1 & -1 & 1 & -1
\end{array}\right),
$$

and $\mathbf{S}=\operatorname{diag}\left(\tau_{0}, \tau_{1}, \ldots, \tau_{8}\right)^{-1}$ is a non-negative diagonal matrix with $\tau_{i}$ being the relaxation time for the $i$ th moment. $f_{e q, i}$ is the discrete equilibrium distribution function given by

$$
f_{e q, i}=\rho w_{i}\left[1+\frac{v_{i} \cdot u}{c_{s}{ }^{2}}+\frac{\left(v_{i} \cdot u\right)^{2}}{2 c_{s}{ }^{4}}+\frac{u \cdot u}{2 c_{s}{ }^{2}}\right],
$$

where $w_{0}=4 / 9, w_{1-4}=1 / 9, w_{5-9}=1 / 36, c_{s}=c / \sqrt{3}$ is the sound speed, and $\rho$ and $u$ are the density and velocity defined as

$$
\rho=\sum_{i} f_{i}, \quad \rho u=\sum_{i} v_{i} f_{i} .
$$

The half-way bounce-back scheme is used to enforce the no-slip boundary condition at solid surface by reflecting VDF from the boundary nodes back into the direction of incidence. ${ }^{52,53}$

\section{INFLUENCE OF THE BEND IN MICRO-CHANNELGAS FLOW}

In this section, we assess the influence of bend on the pressure-driven gas flows in micro-channel. The MFRs are calculated for the Knudsen number ranging from 0.01 to 10 , spanning over the slip and transition flow regimes. The convergence study is performed for both the straight and bent channels to determine the grids in the velocity and spatial spaces. The numbers of discrete points used in the $x$ and $y$ directions are 2,700 and 200, respectively. To capture the discontinuities in the distribution function at large Knudsen numbers, $v_{x}$ and $v_{y}$ are discretized by Eq. (20) with $N_{v}=34$ non-uniform points in each direction. Further refinement of the spatial mesh and discrete velocities would change the results by less than $1 \%$. In addition to the MFR, we are also interested in the pressure distribution, dimensionless velocity magnitude $U=\sqrt{u_{x}^{2}+u_{y}^{2}} / \Delta p$, velocity contours, and the streamlines. 


\section{A. The bend effect on the Knudsen minimum effect}
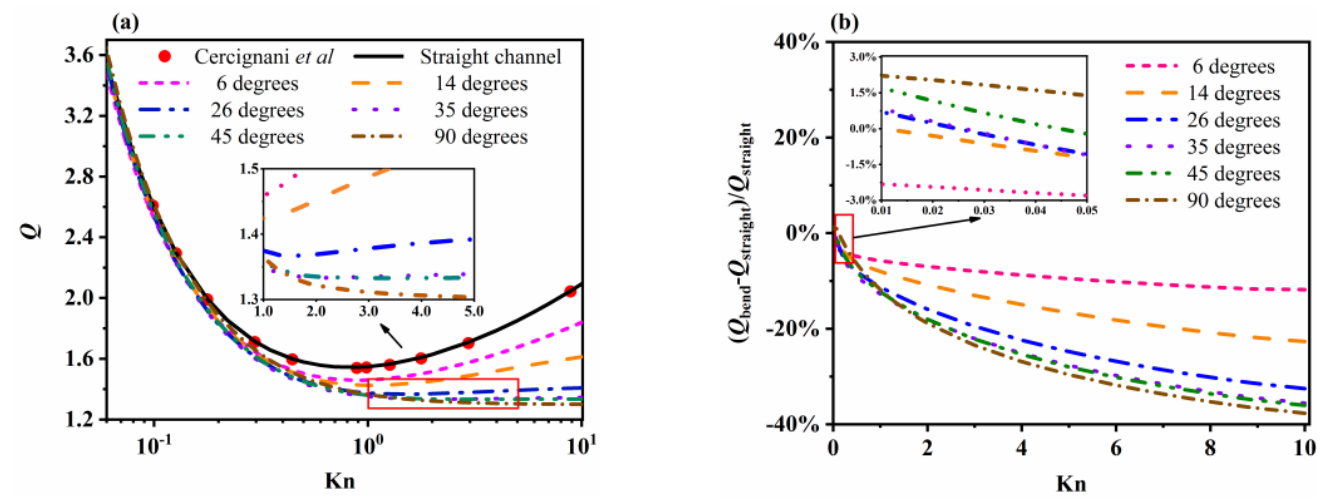

FIG. 2. (a) The dimensionless mass flow rate $Q$ and (b) the relative deviation $\left(Q_{\text {bend }}-Q_{\text {straight }}\right) / Q_{\text {straight }}$ between the straight and bent channels with bend angles $\theta=6,14,26,35,45,90$ degrees. Note that $Q$ is obtained from the DVM solution of the linearized BGK equation and the semi-analytical result from Cercignani, Lampis, and Lorenzani ${ }^{35}$ are labeled as "Cercignani et al".

FIG. 2 shows the change of MFRs with respect to the Knudsen number in the micro-channels with the same total length $L$ but different bend angles $\theta$. As shown in FIG. 2(a), for the straight channel $(\theta=0)$, the MFR decreases to a minimum value at first and then increases slightly with a further increase in the Knudsen number, which is called the Knudsen minimum or Knudsen paradox ${ }^{37}$. The results from Cercignani et $a l^{35}$ are also included as shown in red solid circles. For the MFR in the straight channel, the present DVM solution agrees well with that from Cercignani with less than $0.5 \%$ error.

When gas flows through the bent channels with different bend degrees, one can find that, the MFRs in the bent channel are slightly higher than those in the straight one for small Knudsen numbers. However, when the Knudsen number increases, this slight increment soon disappears and the MFRs drop below the profile in the straight channel significantly. Moreover, the difference between the MFRs in the straight and bent channels enlarges as the bend angle increases. The relative deviation of the MFRs between the bent and straight channels is shown in FIG. 2(b). When $\mathrm{Kn}=0.05,0.5,5.0$, the MFRs are equal to $4.208,1.477$ and 1.303 for the bent channel with $\theta=90^{\circ}$ while for the straight channel the MFRs is $4.149,1.578$ and 1.851 , respectively. This implies that two bends of $90^{\circ}$ cause about $1.39 \%$ increment, $6.43 \%$ and $29.59 \%$ drops in MFRs as compared to that of the straight channel at $\mathrm{Kn}=0.05,0.5$ and 5.0, respectively.

Note that the Knudsen number corresponding to the minimum MFR changes as the bend angle increases. Table I shows the minimum MFR and the corresponding Knudsen number with different bend angles. It is clear that an increase in the bend angle leads to a larger Knudsen number at which the minimum MFR occurs. Specifically, the minimum point appears at $\mathrm{Kn}=0.8$ in the straight channel, but for $\theta=6^{\circ}, \angle \mathrm{U}^{\circ}, 4 \mathcal{J}^{\circ}$ the location shifts to $\mathrm{Kn}=0.90,1.47$ and 3.19, respectively. Furthermore, for the bend angle of $90^{\circ}$, the Knudsen minimum in the whole transition flow regimes even disappears, which means that the MFR monotonically decreases without any increase. This reduction of MFRs indicates that the rarefied effect along with the increase of bend angle leads to an apparent local resistance in the channel jointly. 
TABLE I. Minimum MFRs and corresponding Knudsen numbers for different bend angles, $\theta$

\begin{tabular}{ccc}
\hline \hline Bend angle, $\theta$ & Kn & Mass flow rate \\
\hline $0^{\circ}$ & 0.89 & 1.544 \\
$6^{\circ}$ & 0.90 & 1.457 \\
$14^{\circ}$ & 1.05 & 1.423 \\
$26^{\circ}$ & 1.47 & 1.366 \\
$35^{\circ}$ & 2.10 & 1.334 \\
$45^{\circ}$ & 3.19 & 1.332 \\
$90^{\circ}$ & - & - \\
\hline \hline
\end{tabular}

\section{B. The change of the velocity field under different flow regimes}

To understand how the bend affects the flow field in different flow regimes, we plot the normalized velocity magnitude $U / U_{\text {mean-AAl }}$ along the centerline of the micro-channel with $\theta=90^{\circ}$ (the dotted line A-A1 in FIG. 1) at three different Knudsen numbers in FIG. 3, where $U_{\text {mean- AA1 }}$ is the average velocity magnitude along the centerline A-A1. It can be found that, for the same Knudsen number, the values of $U / U_{\text {mean-AAl }}$ are nearly the same along the channel but only fluctuate violently around the bend corners. Also, the normalized velocity profiles are roughly the same at the bend corner under different Knudsen numbers. The result appears to be consistent with the previous findings in [5,10-12] which argue that bends only affect the flow field locally and the sharp decrease of the normalized velocity reflects the influence of the corner. However, the reason of this sudden change in the velocity magnitude along centerline is mainly due to the fact that the direction of the mainstream deviates from the centerline at the corner. As shown in FIG. 4, the streamlines and the velocity magnitude contour at the corner indicate that the mainstream fluid flows through the bend along the arc direction (direction 1) rather than the centerline (direction 2). Therefore, the fluctuation of the velocity magnitude shown in FIG. 3 could only reflect the deviation between the velocity along the centerline and the one along the mainstream, but not the influence of the bent corner on the flow field.

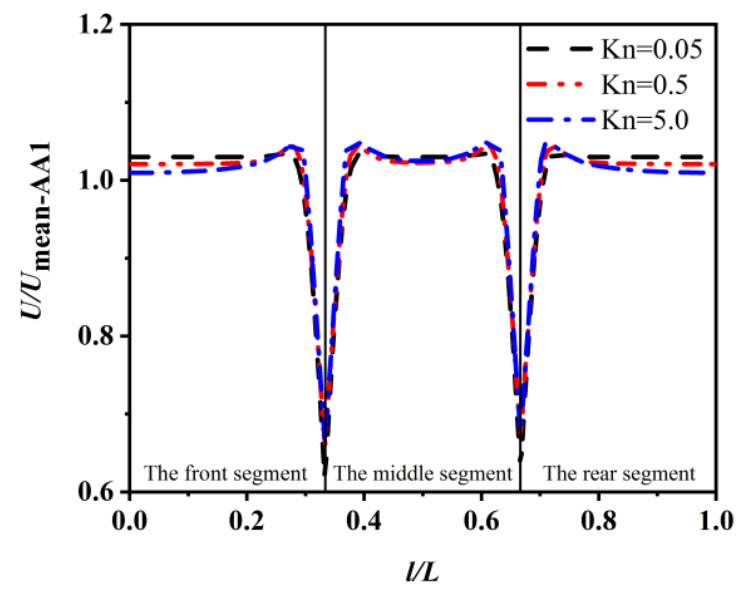

FIG. 3. The normalized velocity magnitude $U / U_{\text {mean-AA } 1}$ along the centerline of the bent channel with $\theta=90$ for $\mathrm{Kn}=0.05,0.5$ and 5.0. $U_{\text {mean-AAl }}$ is the average velocity magnitude along the centerline under the corresponding Knudsen numbers, and $l$ represents the distance from the inlet along the centerline. 


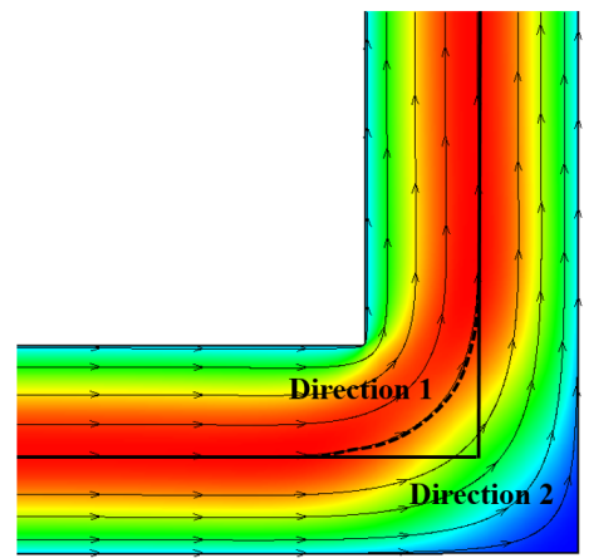

FIG. 4. Streamline and the contour of velocity magnitude at the corner.

We further discuss the velocity magnitude $U$ and the normalized velocity magnitude $U / U_{\text {mean }}$ at different cross sections of the bent channel with $\theta=90^{\circ}$. FIG. 5 shows the velocity magnitude $U$ and the normalized velocity magnitude $U / U_{\text {mean-BB }}$ at $l / L=0.15$ (section B-B in FIG. 1 ), where $U_{\text {mean-BB }}$ is the average velocity magnitude in the B-B section under the corresponding Knudsen numbers. It can be seen that, due to the relatively far distance from the bend corner, the velocity profiles present symmetrical parabolas. As shown in FIG. 5(a), the velocity magnitude in channel is almost the same as that in the straight one at $\mathrm{Kn}=0.05$. However, with the increase of the rarefaction effect, the velocity of the bend channel drops to lower than that of the straight one, and the reduction becomes quite evident at $\mathrm{Kn}=5.0$. Although the difference between the velocity magnitudes becomes significant, the profiles of the normalized velocity magnitude in the bent channel shown in FIG. 5(b) are in good agreement with those in the straight channel under different Knudsen numbers. This indicates that the flow pattern does not change, but only the MFR drops as compared to the straight channel.
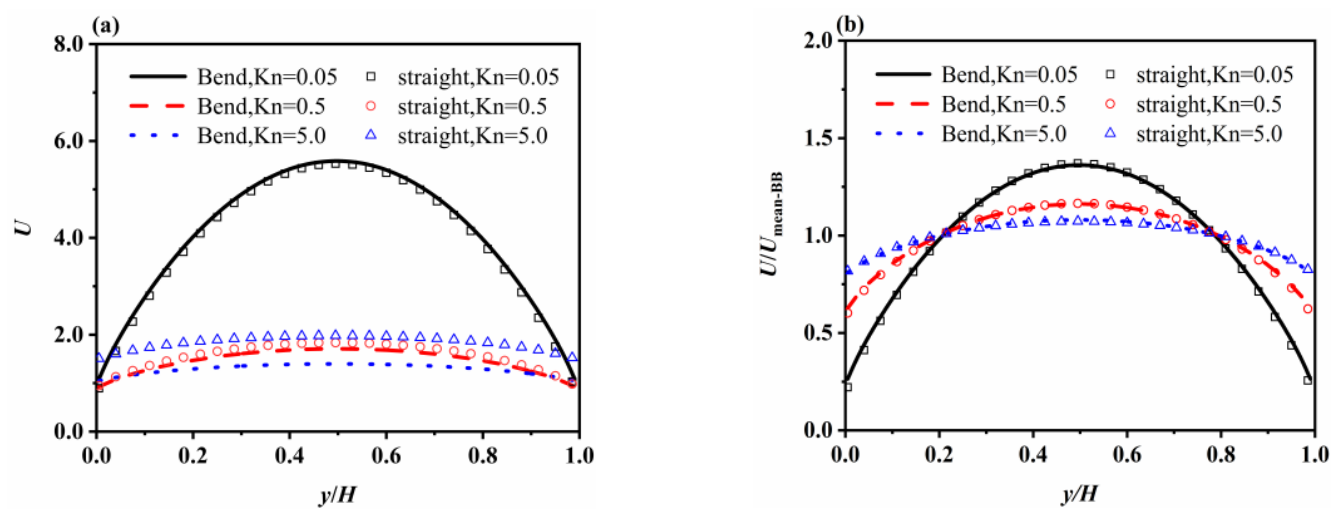

FIG. 5. (a) Velocity magnitude $U$ and (b) normalized velocity magnitude $U / U$ mean-BB at $l / L=0.15$ (section B-B in FIG. 3) in the bent channel with $\theta=90$ for $\mathrm{Kn}=0.05,0.5$ and 5.0. $U_{\text {mean-BB }}$ is the average velocity magnitude in the B-B section under the corresponding Knudsen numbers. $y$ represents the distance from the upper wall along the vertical direction.

FIG. 6 shows the magnitude of the velocity $U$ and the normalized velocity $U / U_{\text {mean-CC }}$ in the cross section at corner (see section C-C in FIG. 1) under three different Knudsen numbers, where $U_{\text {mean-CC }}$ is the average velocity magnitude along the $\mathrm{C}-\mathrm{C}$ section under the corresponding Knudsen numbers. It 
can be found that, due to the constraint of the corner, the velocity profile is no longer a symmetrically parabolic as in the straight channel, and the maximum velocity moves towards the inner wall at $y / H=0$. Moreover, it is noted that the location of the maximum velocity varies at different Knudsen numbers. When $\mathrm{Kn}=0.05$, the maximum velocity appears at $y / H=0.218$, and as the Knudsen number increases to 0.5 and 5.0, the location changes to $y / H=0.090$ and $y / H=0.038$, respectively. This indicates that the gas flow is squeezed into the corner of the inner wall and the velocity near the outer wall (the wall on the side of $y / H=1.0)$ is significantly reduced as the Knudsen number increases.
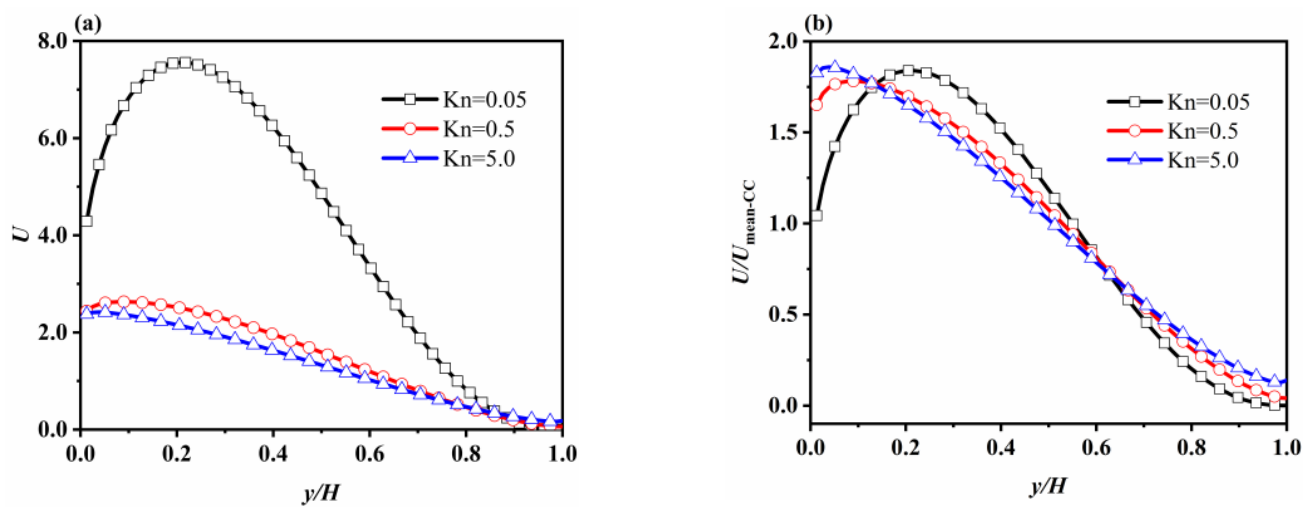

FIG. 6. (a) Velocity magnitude $U$ and (b) normalized velocity magnitude $U / U_{\text {mean-CC }}$ at the center cross section (section C-C in FIG. 1) of the bent channel with $\theta=90$ for $\mathrm{Kn}=0.05,0.5$ and 5.0. $U_{\text {mean-CC }}$ is the average velocity magnitude in the $\mathrm{C}-\mathrm{C}$ section under the corresponding Knudsen numbers. $y$ represents the distance from the inner wall along the section.

\section{The enhancement and reduction of MFR at different Knudsen numbers}

As shown in FIG. 2, in small Knudsen number regime, the MFR through the bent channel is higher than that in the straight one. Some researchers tried to find out the underlying reasons in detail. Agrawal et al. attributed this slight increment in MFR to the numerical error, ${ }^{10}$ while Rovenskaya et $a l .{ }^{5}$ and White et al. ${ }^{12}$ explained that this is attributed to the increase of the local Knudsen at the inner corner tip, which leads to the increase of the local slip velocity and decrease of the local shear stress. To investigate gas flow in the near continuum flow, we use Multiple-Relaxation-Time lattice Boltzmann method (MRT-LBM) to calculate same problem with zero velocity-slip boundary condition in incompressible limit. By using the MRT-LBM to calculate the gas flow through the bent channel in the continuum flow regime, we also find a slight increase of the MFR in the bent channel compared to the straight one. As shown in FIG.7, the values of $Q_{\text {bend }} / Q_{\text {straight }}$ are equals to $1.031,1.022$ and 1.014 when $\mathrm{Kn} \approx 0$ (MRT-LBM result), 0.01 and 0.05 (DVM results), respectively. To further elucidate the enhancement of MFR in the bent channel, we also plot the relative pressure difference $\left(p_{\text {bend }}-p_{\text {straight }}\right) / p_{\text {in }}$ along the center line direction in FIG. 8 , where $p_{\text {in }}$ is the inlet pressure, while $p_{\text {bend }}$ and $p_{\text {straight }}$ are the pressures along the centerlines of the bend and straight channels, respectively. When the Knudsen number is relatively small $(\mathrm{Kn}=0.05)$, the pressure before the corner is slightly lower than that in the straight channel, while the pressure after the corner is slightly higher. This pressure distribution is quite similar to the one in the channel which contains expansion part ${ }^{54}$. Therefore, the increased cross-section at the corner plays a role like an expansion tube, which leads to the result that the MFR is larger than that in the straight channel. So this slight increment in MFR is caused by the increasing cross-section at the corner instead of the rarefied gas effect. 


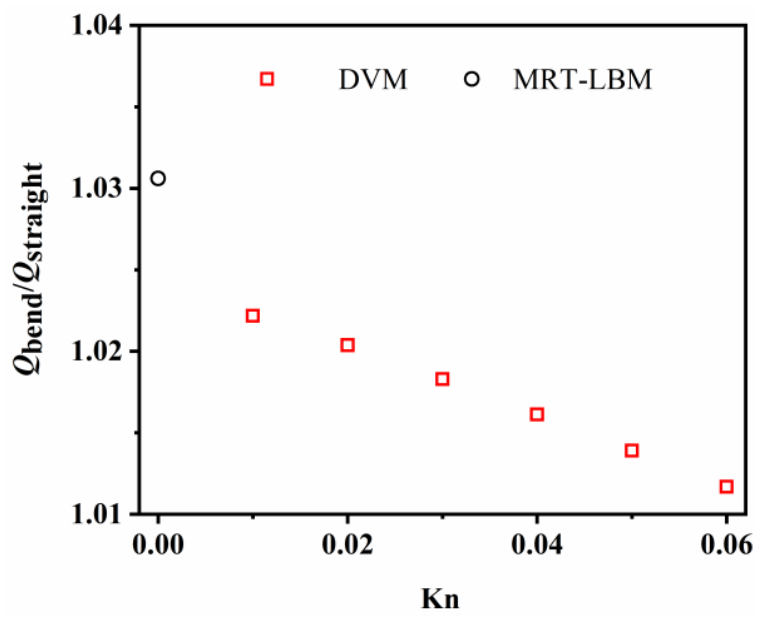

FIG. 7. The ratio of $Q_{\text {bend }}$ and $Q_{\text {straight }}$ in the near continuum and slip flow regimes.

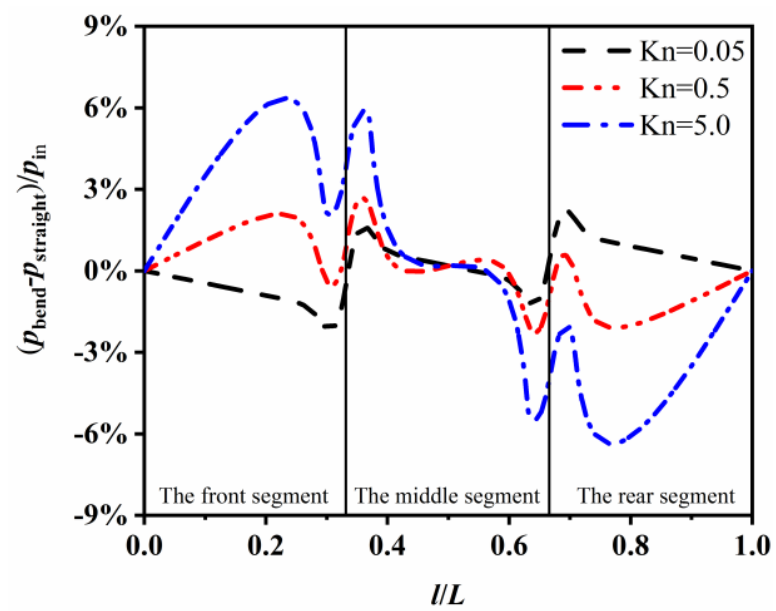

FIG. 8. The pressure difference $\left(p_{\text {bend }}-p_{\text {straight }}\right) / p_{\text {in }}$ along the centerline for $\mathrm{Kn}=0.05,0.5$ and $5.0 . l$ represents the distance from the inlet along the centerline, $p_{\text {in }}$ is the inlet pressure, while $p_{\text {bend }}$ and $p_{\text {straight }}$ are the pressures along the centerlines of the bent and straight channels, respectively.

As the Knudsen number increases, the rarefaction effect tends to dominate, which triggers a variety of counter-intuitive phenomena. One of them is called the Knudsen minimum where the dimensionless MFR in Poiseuille flow could increase when the gas pressure decreases. ${ }^{4}$ This is a paradoxical behavior because, based on the Navier-Stokes equations, one would expect the mass flux to decrease with the increasing Knudsen number. Whereas if the MFR in straight long channel is plotted over the Knudsen number, a distinct minimum is observed around $\mathrm{Kn}=0.8$. The balance of two competing effects could explain the mechanism behind this intuitively: on the one hand, the imposed pressure gradient gradually penetrates the bulk flow field through gas-gas and gas-surface collisions. As the Knudsen number rises, the interaction between the gas molecules are weakened, which could be characterized as the increase of viscosity qualitatively and thus the MFRs drops; on the other hand, as rarefaction effect becomes evident, the impact of the collisions between gas molecules and solid surfaces gradually become dominant. Thus the slip velocity in the Knudsen layer (the gas layer of a few mean free paths to the wall) becomes large, which eventually helps to increase the MFR. The 
combination of the two mechanisms induces the existence of the Knudsen minimum in the Poiseuille flow inside straight channel.

However, when there is a corner in the channel, the collision and rebound direction between the gas and the wall changes. This result in the slip velocity inside the Knudsen layer is no longer parallel to the main flow direction, which may disturb and even slow down the mainstream gas flow. To further reveal the reason for the change of the Knudsen minimum effect, we plot the normalized velocity magnitude $U / U_{\max }$ and the flow streamline in the bent channel with $\theta=90^{\circ}$ at three different Knudsen numbers in Fig. 9, where $U_{\max }$ is chosen as the maximum magnitude of the velocity under the corresponding Knudsen number in the whole flow field. It is seen that the flow fields are similar under different Knudsen numbers except for the contour near the corner. The smooth streamline through the corner indicates that no vortices or flow separations happen for such low-speed flows. ${ }^{38,55}$ For the sake of convenient comparison, FIG. 10 illustrates the contour of the normalized velocity magnitude $U / U_{\max }$ at the first corner by using the maximum magnitude of velocity as the reference. It is shown that the contour line of the velocity at the bend possesses concave, as if the flow is squeezed by the corner. This effect aggravates with the increase of Knudsen number, which indicates that with the increase of the rarefaction effect, the disturbance caused by the corner gradually rises, resulting in a larger area of low velocity at the corner. What's more, Fig. 7 shows that the pressure before the corner increases as compared to that in the straight channel while the pressure after the corner drops, which is very similar to the pressure distribution of the fluid through the narrowing tube or throttle. ${ }^{54}$ Under this circumstance, the corner plays a role similar to a "valve", which results in a decrease of the effective flow section. The main cause of this "rarefaction throttling effect" should be attributed to the sudden change of the mainstream direction imposed by the bend corner.

It should be noted that, even though we carefully selected the aspect ratio of the bent channel $L / H=13.5$ in the two-dimensional simulations according to the previous literature $[5,40,41]$ in order to maintain reasonable results in the three-dimensional cases, there might be different behavior due to greater wall interactions in the corners of the bend under three-dimensional conditions, which may lead to more pronounced Rarefaction Throttling effect.

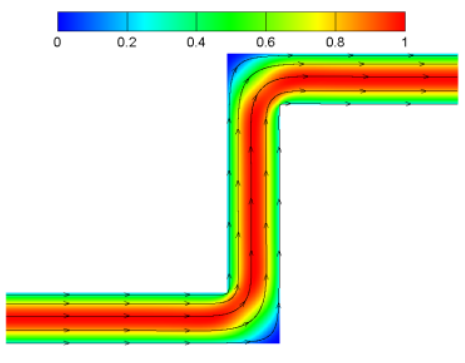

(a) $\mathrm{Kn}=\mathbf{0 . 0 5}$

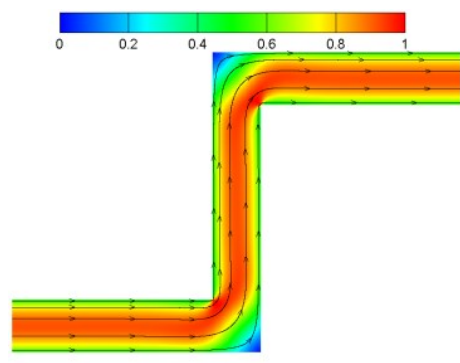

(b) $\mathrm{Kn}=0.5$

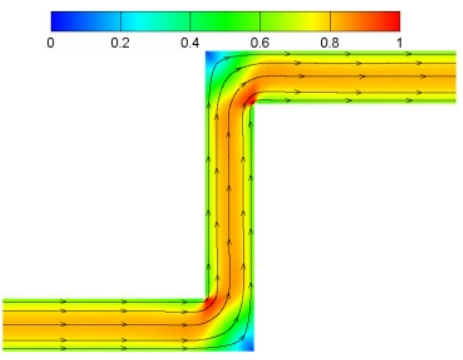

(c) $\mathrm{Kn}=5.0$

FIG. 9. Contours of the normalized velocity magnitude $\left(U / U_{\max }\right)$ and the flow streamlines for the bent channel when (a) $\mathrm{Kn}=0.05$, (b) $\mathrm{Kn}=0.5$, (c) $\mathrm{Kn}=5.0$. $U_{\max }$ is chosen as the maximum velocity magnitude under the corresponding Knudsen number. 

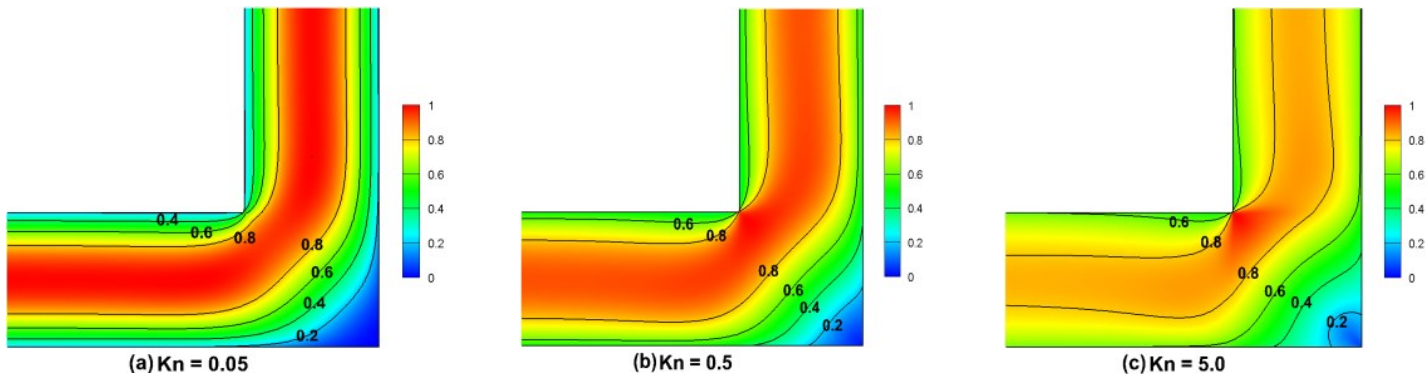

FIG. 10. Contours of the normalized velocity magnitude $U / U_{\max }$ at the first corner when (a) $\mathrm{Kn}=0.05$, (b) $\mathrm{Kn}=0.5$, (c) $\mathrm{Kn}=5.0$. The lines marked with $0.2,0.4,0.6$, and 0.8 represent velocity contour lines of the $20 \%, 40 \%, 60 \%$, and $80 \%$ of the maximum velocity magnitude, respectively.

\section{Effect of the chamfering in bent channel}

To give guidance for the design of the channel, we add a chamfer of $45^{\circ}$ to the corner of bend. FIG. 11 shows the contour of the normalized velocity magnitude $U / U_{\max }$ in the $90^{\circ}$ bent channel with chamfer. It is found that the chamfer makes the gas turn smoothly at the bend, and the area of the local low-velocity area is no longer concentrated near the outer wall of the corner. However, the comparison of the MFRs in channels before and after chamfering (shown in FIG. 12) indicates that the MFR only increases slightly after chamfering, and the amount of increase gradually drops with the increasing Knudsen number. This is consistent with the result shown in FIG. 2, where the change of the bend angle from $90^{\circ}$ to $45^{\circ}$ only causes an increase in the MFR of less than $1.7 \%$.

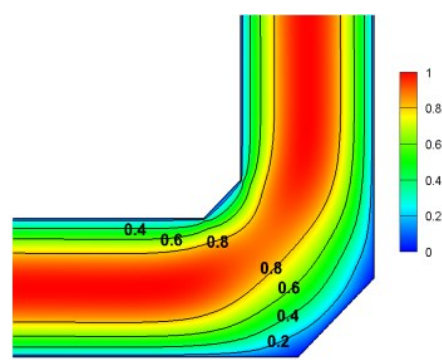

(a) $\mathrm{Kn}=0.05$

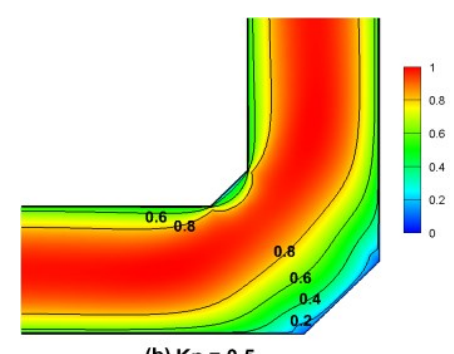

(b) $\mathrm{Kn}=0.5$

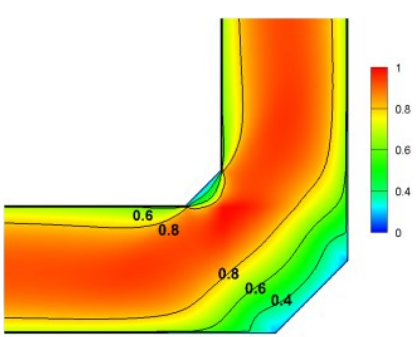

(c) $\mathrm{Kn}=5.0$

FIG. 11. Contours of the normalized velocity magnitude at the chamfered corner. The lines marked with $0.2,0.4,0.6$ and 0.8 represent velocity contour lines of the $20 \%, 40 \%, 60 \%$ and $80 \%$ maximum velocity magnitude, respectively. 


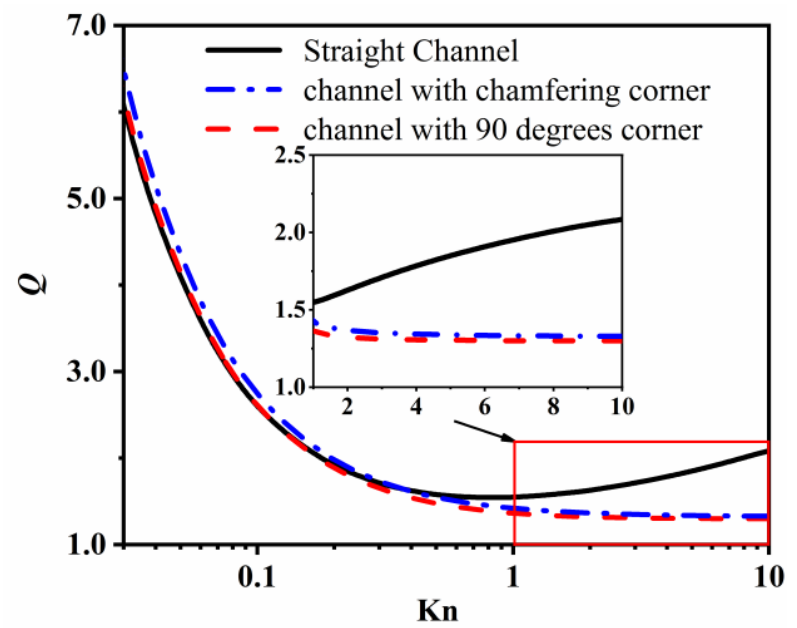

FIG. 12. The mass flow rate $Q$ in the straight channel, channel with chamfering corner and channel with 90 degrees corner.

\section{E. Effect of the tangential momentum accommodation coefficient in bent channel}

Finally, we change the TMAC $\alpha$ to examine the effect of different wall conditions on gas flow in micro-channel with bend. FIG. 13(a) shows the MFR in the straight channel under different $\alpha$. It can be found that the present DVM solutions agree well with those from Cercignani et al. ${ }^{35}$ with relative error less than $0.5 \%$. FIG. 13(b) shows the results for the MFR in the channel with bend angle of $90^{\circ}$ under different $\alpha$. The MFR increases with the drop of $\alpha$ at the same Knudsen number. This is because $\alpha$ represents the proportion of the diffusive reflection on the wall. As $\alpha$ decreases, the proportion of diffuse reflectance decreases and the specular reflectance increases, so that the velocity slip gradually becomes significant (note that the slip coefficient is roughly proportional to $(2-\alpha) / \alpha)$. The ratio of MFRs in the channel with bend and the one in the straight channel $Q_{\text {bend }} / Q_{\text {straight }}$ at different TMACs are given in FIG. 14. It can be seen that, for a fixed value of $\alpha$, the MFR ratios decrease with the increase of Knudsen number. The smaller the TMAC is, the more rapidly the MFR ration drops as Kn increases. For a fixed Knudsen number, the MFR ratio drops as the TMAC decreases. This indicates that the slip velocity at the corner is a vital factor in the generation of the "throttling effect", and with small TMAC, the "throttling effect" could become significant even at relatively small Knudsen numbers.
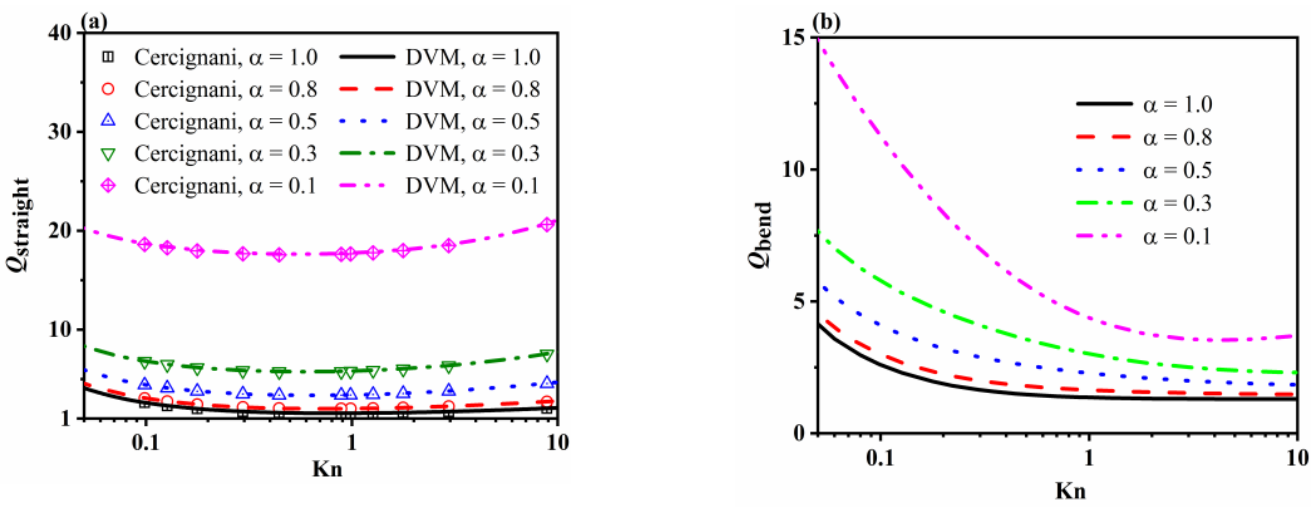
FIG. 13. (a) The dimensionless mass flow rate $Q_{\text {straight }}$ in the straight channel, and (b) the dimensionless mass flow rate $Q_{\text {bend }}$ in bent channel with bend angle of $90^{\circ}$ under different tangential momentum accommodation coefficients $\alpha$.

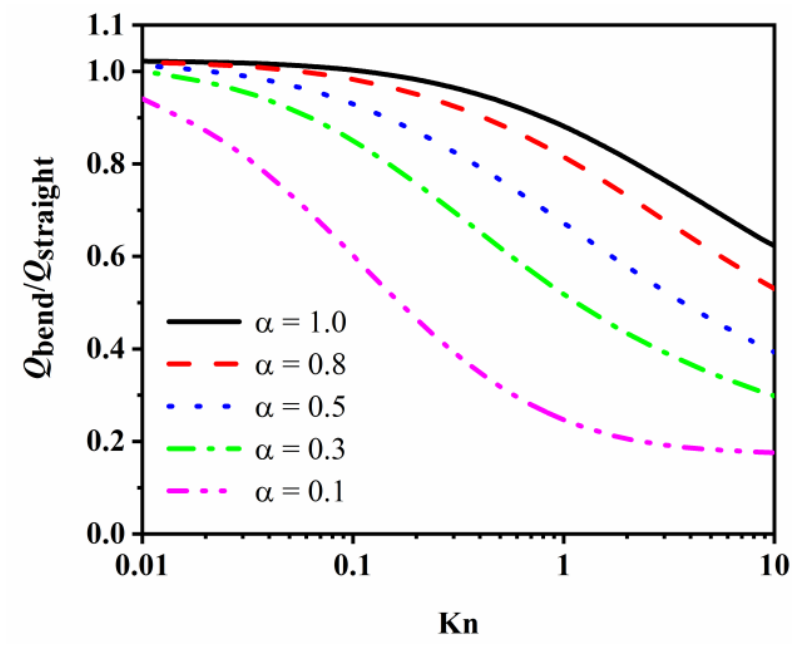

FIG. 14. The ratio of the MFR in the channel with bend and the one in the straight channel $Q_{\text {straight }} / Q_{\text {bend }}$ under different tangential momentum accommodation coefficients $\alpha$.

\section{v. CONCLUSIONS}

In summary, based on the linearized Bhatnager-Gross-Krook equation, we have investigated the behavior of a two-dimensional pressure-driven gas flow through a micro-channel with two bends. The mass flow rates (MFR) of the gas flows in the bent micro-channel with different bend angles are obtained. Our results show that the effects of the corners on the flow field depend on Knudsen numbers. The main findings could be summarized as follows.

1. At small Knudsen numbers, the dimensionless MFR through the bend is slightly higher than that in the straight channel. Since this phenomenon is also found in the continuum flow regime by using MRT-LBM method that solves the Navier-Stokes equation with zero velocity-slip boundary condition, for the first time we find that the slight increase of MFR is not due to the rarefaction gas effect but to the increase in cross-section induced by the corner.

2. As the Knudsen number increases, the MFR in the bent channels starts to decline and the amount of reduction increases significantly compared to the straight channel. The increase of the bend angle would also reduce MFR under the same Knudsen number. Besides, the Knudsen number corresponding to the minimum location of MFR gradually increases with the bend angle, and even disappears in the slip and transition flow regimes when the bend angle reaches 90 degrees. The distributions of normalized velocity at different cross sections show that, as the Knudsen number increases, the zone of low velocity at the bend gradually expands and the gas flows are "squeezed" into the inner corner, which is very similar to the throttling effect.

3. Moreover, the MFR through the micro-channel with chamfer corner is calculated. Although the low-velocity area is not as concentrated as the $90^{\circ}$ corner, the reduction of MFR is not improved apparently.

4. The decrease in tangential momentum accommodation coefficient would enhance the slip 
velocity on the wall, which could exacerbate the rarefaction throttling effect and allow it to occur at very low Knudsen numbers.

Finally, it is noted that the bent channel should not only be considered as itself, but also a very basic component of complex geometry. Understanding this rarefaction throttling effect would help to evaluate the MFRs affected by the complex geometry under low-velocity and rarefied circumstances, especially in a quite tortuous geometry such as micron-sized chips and shale rocks.

\section{ACKNOWLEDGMENTS}

This work was financially supported by the National Natural Science Foundation of China under grant number 51721004, the joint project from the Royal Society of Edinburgh and National Natural Science Foundation of China under Grant No. 51711530130, the Carnegie Research Incentive Grant for the Universities in Scotland and the 111 Project under grant number B16038.

${ }^{1}$ C.M. Ho and Y.C. Tai, "Micro-Electro-Mechanical-Systems ( Mems ) and Fluid,” Annu. Rev. Fluid Mech. 30, 579 (1998).

${ }^{2}$ D.J. Beebe, G.A. Mensing, and G.M. Walker, "Physics and applications of microfluidics in biology,” Annu. Rev. Biomed. Eng. 4, 261 (2002).

${ }^{3}$ T. Thorsen, S.J. Maerkl, and S.R. Quake, “Microfluidic Large-Scale Integration,” Science. 298, 580 (2002).

${ }^{4}$ Z. Xu, H. Liu, and A.J. Valocchi, "Lattice Boltzmann simulation of immiscible two-phase flow with capillary valve effect in porous media,” Water Resour. Res. 53, 3770 (2017).

${ }^{5}$ O.I. Rovenskaya, “Computational study of 3D rarefied gas flow in microchannel with $90^{\circ}$ bend,” Eur. J. Mech. B/Fluids 59, 7 (2016).

${ }^{6}$ F. Sharipov and V. Seleznev, "Data on Internal Rarefied Gas Flows,” J. Phys. Chem. Ref. Data 27, 657 (1998).

${ }^{7}$ S. Varoutis, S. Naris, V. Hauer, C. Day, and D. Valougeorgis, “Computational and experimental study of gas flows through long channels of various cross sections in the whole range of the Knudsen number," J. Vac. Sci. Technol. A Vacuum, Surfaces, Film. 27.1, 89 (2009).

${ }^{8}$ T. Ewart, P. Perrier, I.A. Graur, and J.G. Méolans, "Mass Flow Rate Measurements in a Micro Channel: from Hydrodynamic to Free Molecular Regime,” J. Fluid Mech. 584, 337 (2007).

${ }^{9}$ G.H. Tang, Z. Li, Y.L. He, and W.Q. Tao, "Experimental study of compressibility, roughness and rarefaction influences on microchannel flow,” Int. J. Heat Mass Transf. 50, 2282 (2007).

${ }^{10}$ A. Agrawal, L. Djenidi, and A. Agrawal, "Simulation of gas flow in microchannels with a single $90^{\circ}$ bend," Comput. Fluids 38, 1629 (2009).

${ }^{11}$ O. Rovenskaya and G. Croce, "Numerical Analysis of Rarefaction and Compressibility Effects in Bent Microchannels," in 8th Int. Conf. Nanochannels, Microchannels, Minichannels Collocated with 3rd Jt. US-European Fluids Eng. Summer Meet. (ASME, 2010, ICNMM, 2010), pp. 451-458.

${ }^{12}$ C. White, M.K. Borg, T.J. Scanlon, and J.M. Reese, “A DSMC investigation of gas flows in micro-channels with bends,” Comput. Fluids 71, 261 (2013).

${ }^{13}$ A. Karniadakis GEM, A. Beskok A, and R. Gad-el-Hak M, "Micro flows: fundamentals and simulation,” Appl. Mech. Rev. 55, B76 (2002). 
${ }^{14}$ G.H. Tang, W.Q. Tao, and Y.L. He, “Gas slippage effect on microscale porous flow using the lattice Boltzmann method,” Phys. Rev. E 72, 056301 (2005)

${ }^{15}$ Y. Sone, T. Ohwada, and K. Aoki, "Temperature jump and Knudsen layer in a rarefied gas over a plane wall: numerical analysis of the linearized Boltzmann equation for hard-sphere molecules," Phys. Fluids A 1, 363-370 (1989).

${ }^{16}$ W. Steckelmacher, "Knudsen flow 75 years on - The current state of the art for flow of rarefied gases in tubes and systems," Reports Prog. Phys. 49, 1083 (1986).

${ }^{17}$ O. Reynolds, "The dimensional properties of matter in the gaseous state," Nature 19, 435 (1879).

${ }^{18}$ S.Y.K. Lee, M. Wong, and Y. Zohar, “Gas flow in microchannels with bends,” J. Micromech. Microeng. 11, 635 (2001).

${ }^{19}$ X. Li, W.Y. Lee, M. Wong, and Y. Zohar, "Gas flow in constriction microdevices," Sensors Actuators, A Phys. 83, 277 (2000).

${ }^{20}$ V. Varade, A. Agrawal, S. V. Prabhu, and A.M. Pradeep, "Early onset of flow separation with rarefied gas flowing in a $90^{\circ}$ bend tube,” Exp. Therm. Fluid Sci. 66, 221 (2015).

${ }^{21}$ S. Chapman, T.G. Cowling, and D. Park, “The mathematical theory of non-uniform gases,” Am. J. Phys. 30, 389 (1962)

${ }^{22}$ G.A. Bird, Molecular Gas Dynamics and the Direct Simulation of Gas Flows (Oxford University Press, 1994).

${ }^{23}$ M. Wang and Z. Li, "Simulations for gas flows in micro-geometries using the direct simulation Monte Carlo method,” Int. J. Heat Fluid Flow 25, 975 (2004).

${ }^{24}$ N.K. Kulakarni, K. Shterev, and S.K. Stefanov, "Effects of finite distance between a pair of opposite transversal dimensions in microchannel configurations: DSMC analysis in transitional regime,” Int. J. Heat Mass Transf. 85, 568 (2015)

${ }^{25}$ C. Cai, I.D. Boyd, J. Fan, and G. V. Candler, “Direct Simulation Methods for Low-Speed Microchannel Flows,” J. Thermophys. Heat Transf. 14, $368(2000)$

${ }^{26}$ V. V. Aristov, Direct Methods for Solving the Boltzmann Equation and Study of Nonequilibrium Flows. (Springer, Netherlands, 2001).

${ }^{27}$ C.K. Chu, "Kinetic-theoretic description of the formation of a shock wave," Phys. Fluids 8, 12 (1965)

${ }^{28}$ J.Y. Yang and J.C. Huang, "Rarefied Flow Computations Using Nonlinear Model Boltzmann Equations,” J. Comput. Phys. 120, $323(1995)$

${ }^{29}$ F. Sharipov, "Rarefied gas flow through a slit. Influence of the boundary condition,” Phys. Fluids 8, 262 (1996).

${ }^{30} \mathrm{~L}$. Wu, J.M. Reese, and Y. Zhang, "Solving the Boltzmann equation deterministically by the fast spectral method: application to gas micro flows,” J. Fluid Mech. 746, 53 (2014).

${ }^{31}$ S. Taguchi and K. Aoki, "Rarefied gas flow around a sharp edge induced by a temperature field,” J. Fluid Mech. 694, 191 (2012).

${ }^{32}$ P.L. Bhatnagar, E.P. Gross, and M. Krook, “A model for collision processes in gases. I. Small amplitude processes in charged and neutral one-component systems," Phys. Rev. 94, 511 (1954).

${ }^{33}$ W. Su, H. Liu, Y. Zhang, and L. Wu, "Rarefaction Cloaking: Influence of the Fractal Rough Surface in Gas Slider Bearings," Phys. Fluids 29, (2017).

${ }^{34} \mathrm{~W}$. Su, S. Lindsay, H. Liu, and L. Wu, "Comparative study of the discrete velocity and lattice Boltzmann methods for rarefied gas flows through regular channels," Phys. Rev. E 96, (2017).

${ }^{35}$ C. Cercignani, M. Lampis, and S. Lorenzani, "Plane Poiseuille flow with symmetric and non-symmetric gas-wall interactions," Transp. Theory Stat. Phys. 33, 545 (2004) 
${ }^{36}$ X. J. Gu, D. R. Emerson, and G. H. Tang, "Analysis of the slip coefficient and defect velocity in the Knudsen layer of a rarefied gas using the linearized moment equations,” Phys. Rev. E 81, 016313 (2010).

${ }^{37}$ M. Knudsen, "Die gesetze der molekularstrmung und der innerenreibungsstrmung der gasedurchrhren," Ann. Phys. 333, 75 (1909).

${ }^{38}$ F. Sharipov and I.A. Graur, "Rarefied gas flow through a zigzag channel,” Vacuum 86, 1778 (2012).

${ }^{39} \mathrm{~T}$. Veijola, H. Kuisma, and J. Lahdenperä, “The influence of gas-surface interaction on gas-film damping in a siliconaccelerometer," Sensors Actuators A Phys. 66, 83 (1998).

${ }^{40}$ A. Agrawal and A. Agrawal, "Three-dimensional simulation of gaseous slip flow in different aspect ratio microducts," Phys. Fluids 18,579-56 (2006).

${ }^{41}$ C.E. Zhen, Z.C. Hong, Y.J. Lin, and N.T. Hong, "Comparison of 3-D and 2-D DSMC heat transfer calculations of low-speed short microchannel flows,” Numer. Heat Transf. Part A Appl. 52,239-250 (2007).

${ }^{42}$ Stefanov, S. K., N. K. Kulakarni, and K. S. Shterev, "Modeling of gas flows through microchannel configurations," in International Conference for Promoting the Application of Mathematics in Technical \& Natural Sciences-amitans American Institute of Physics. (AMiTaNS, 2013), pp.59-67.

${ }^{43}$ J. C. Maxwell, "On stresses in rarefied gases arising from inequalities of temperature,” Philos. Trans. R. Soc. London 170, 231-256 (1879).

${ }^{44}$ W. M. Zhang, G. Meng, and X. Wei, “A review on slip models for gas microflows,” Microfluid. Nanofluidics13, 845 (2012).

${ }^{45}$ C. Cercignani, The Boltzmann Equation and Its Applications (Springer-Verlag, New York, 1988).

${ }^{46}$ Z. Guo, C. Zheng, and B. Shi,” Lattice Boltzmann equation with multiple effective relaxation times for gaseous microscale flow,” Phys. Rev. E. 77, (2008).

${ }^{47}$ H. Liu, Q. Kang, C.R. Leonardi, S. Schmieschek, A. Narváez, B.D. Jones, J.R. Williams, A.J. Valocchi, and J. Harting, "Multiphase lattice Boltzmann simulations for porous media applications," Comput. Geosci. 20, 777-805 (2015).

${ }^{48}$ H. Liu, A.J. Valocchi, and Q. Kang, “Three-dimensional lattice Boltzmann model for immiscible two-phase flow simulations,” Phys. Rev. E 85, 046309 (2012).

${ }^{49}$ H. Liu and Y. Zhang,” Droplet formation in microfluidic cross-junctions,” Phys. Fluids 23, (2011).

${ }^{50} \mathrm{P}$. Lallemand and L. Luo,” Theory of the lattice Boltzmann method: Dispersion, dissipation, isotropy, Galilean invariance, and stability, "Phys. Rev. E 61, 6546-6562 (2000).

${ }^{51}$ Z. Guo and C. Zheng, "Analysis of lattice Boltzmann equation for microscale gas flows: Relaxation times, boundary conditions and the Knudsen layer,” Int. J. Comut. Fluid Dyn. 22,465-473 (2008).

${ }^{52}$ I. Ginzbourg and P.M. Adler, "Boundary flow condition analysis for the three-dimensional lattice Boltzmann model," J. Phys. II France 4, 191 (1994).

${ }^{53}$ X. He, Q. Zou, L.S. Luo, and M. Dembo, "simple flows and analysis of nonslip boundary conditions for the lattice Boltzmann BGK model,” J. Stat. Phys. 87, 115 (1997).

${ }^{54}$ A. Agrawal, L. Djenidi, and R.A. Antonia, "Simulation of gas flow in microchannels with a sudden expansion or contraction,"

J. Fluid Mech. 530, 135 (2005).

${ }^{55}$ F. Sharipov, "Non-isothermal rarefied gas flow through a slit,” Phys. Fluids 9, 1804 (1997). 